\title{
A Cloud Adoption Decision Support Model Using Influence Diagrams
}

\author{
Andreas Christoforou and Andreas S. Andreou \\ Department of Electrical Engineering / Computer Engineering and Informatics, \\ Cyprus University of Technology \\ ax.christoforouledu.cut.ac.cy, andreas.andreoulcut.ac.cy
}

\begin{abstract}
Cloud Computing has become nowadays a significant field of Information and Communication Technology (ICT), and this has led many organizations moving their computing operations to the Cloud. Decision makers are facing strong challenges when assessing the feasibility of the adoption of Cloud Computing for their organizations. The decision to adopt Cloud services falls within the category of complex and difficult to model real-world problems. In this paper we propose an approach based on Influence Diagrams modeling, aiming to support the Cloud adoption decision process. The developed ID model combines a number of factors which were identified through litterature review and input received from field experts. The proposed approach is validated against four experimental cases, two realistic and two real-world, and its performance proved to be highly capable of estimating and predicting correctly the right decision.
\end{abstract}

Keywords: Influence Diagrams, Cloud Adoption, Decision Support.

\section{Introduction}

Cloud Computing is changing the whole perspective with which we understand computing today. The adoption of Cloud Computing is still a major challenge for organizations daily producing and processing information in the context of their working activities. A constantly increasingly number of companies include Cloud Computing in their short or long term planning since sufficient number of services that are available on the Cloud has surpassed infancy and appears to be quite mature and attractive. Many of the major software developers or service providers have already turned their strategy towards Cloud services mostly targeting at increasing their market share. On one hand companies-customers need to consider the benefits, risks and effects of Cloud Computing on their organization in order to proceed with adopting and using such services, and on the other hand Cloud Computing providers need to be fully aware of customers' concerns and understand their needs so that they can adjust and fit their services accordingly.

Although in recent years the research community has increasingly been interested in this field, a review of the literature on Cloud Computing, and especially on Cloud adoption, revealed that there are yet no mature techniques or toolkits to support the decision making process for adopting Cloud services on behalf of customers. 
The ambiguity and uncertainty often surrounding the Cloud adoption, deriving of the multiple, conflicting factors in combination with simplistic assumptions, makes Cloud adoption a highly complex process that cannot be satisfied using classical and linear methods. Furthermore, the extremely fast-moving nature of the Cloud Computing environment changes, both to supply and demand, shows how difficult it may be for any procedure to assist the decision making process timely and correctly. This is the reason why a framework or model which supports Cloud Computing adoption should be quite flexible and dynamically adaptable.

In this paper we propose a methodology based on Influence Diagrams (IDs) which was used to set-up a successful decision model to support Cloud adoption. The model was constructed in a systematic manner: Firstly we performed a study of the most recent and relevant literature on Cloud Computing and particularly on Cloud adoption, through which we identified all possible factors that influence the final Cloud adoption decision. Next, based on the result of this study, we proceeded to categorized those factors, and build and distribute a questionnaire to a group of experts so as to capture their knowledge and expertise as regards approving the list of factors already identified. In addition, the experts were called to define the relation of each factor to Cloud adoption and a corresponding weight on a Likert scale. Finally and using the collected information we developed a novel model based on Influence Diagrams that answers the question "Adopt Cloud Services?" under the current state of the offered service and the associated factors describing each customer's particular situation at the moment of decision.

The rest of the paper is organized as follows: Section 2 presents related work in the area of Cloud Computing adoption based on the existing literature. Section 3 makes a brief description of the theory of Influence Diagrams, while section 4 introduces the Cloud adoption modeling process and discusses and analyses the corresponding experimental results. Finally, section 5 provides our conclusions and suggestions for future research steps.

\section{Related Work}

Among many definitions of Cloud Computing, a working definition that has been published by the US National Institute of Standards and Technology (NIST) [8], captured the most common agreed aspects. NIST defines Cloud Computing as "a model for enabling ubiquitous, convenient, on-demand network access to a shared pool of configurable computing resources (e.g., networks, servers, storage, applications and services) that can be rapidly provisioned and released with minimal management effort or service provider interaction." This Cloud model promotes availability and is composed of five essential characteristics, three service models and four deployment models as follows:

- Characteristics: on-demand self-service, broad network access, resource pooling, rapid elasticity and measured service.

- $\quad$ Service models: Software as a Service (SaaS), Platform as a Service (PaaS) and Infrastructure as a Service (IaaS).

- Deployment models: private Clouds, community Clouds, public Clouds and hybrid Clouds. 
Although it is generally recognized that the adoption of Cloud Services can offer substantial benefits, many organizations are still reluctant to proceed with it. There is a variety of factors that may influence Cloud adoption and it is quite important to properly identify and analyze them aiming to assist customers in taking the right decision. Equally important in this study, from the vendors' point of view, is to define which factors should possibly change so as to revert a current negative adoption decision. Our research is mainly focused on the SaaS model investigating both the Cloud providers' and the customers' point of view.

An investigation of the current literature revealed a relatively small number of papers discussing Cloud adoption from the perspective of decision making and also current feasibility approaches fall short in terms of decision making to determine the right decision. We introduce a summary of these studies, examining the contribution of each work to the decision making problem. In [9] Khajeh-Hosseini et al. presented a Cloud adoption toolkit which provides a framework to support decision makers in identifying their concerns and match them with the appropriate techniques that can be used to address them. Kim et al. [10] examined various issues that impede rapid adoption of Cloud Computing such as cost, compliance and performance. Wu in [6] attempted to contribute to the development of an explorative model that extends the practical applications of combining Technology Acceptance Model (TAM) related theories with additional essential constructs such as marketing effort, security and trust, in order to provide a useful framework for decision makers to assess the issue of SaaS adoption and for SaaS providers to become sensitive to the needs of users. Additionally, $\mathrm{Wu}$ [7] explored the significant factors affecting the adoption of SaaS by proposing an analytical framework containing two approaches: the Technology Acceptance Model (TAM) related theories and the Rough Set Theory (RST) data mining. In [11], a solution framework is proposed that employs a modified approach proposed in the 70s named DEMATEL [12] to cluster a number of criteria (perceived benefits and perceived risks) into a cause group and an effect group respectively, presenting also a successful case study. Even though all of the above techniques contribute a significant piece to this new open research field, they may be classified as "traditional", single layer approaches which examine only a specific part of the problem.

Techniques that use IDs in modeling decision process seem to improve the way the problem is approached by offering various strong benefits. IDs offer flexibility representing many dependencies between factors and manage to represent a highly complex problem in a human understandable way. Also, IDs allow interaction of experts through execution of the model with input combinations thus utilizing their expertise in order to calibrate the model and achieve reasonable and helpful answers.

\section{$3 \quad$ Influence Diagrams}

An Influence Diagram (ID) [2] is a general, abstract, intuitive modeling tool that is nonetheless mathematically precise [1]. IDs are directed graph networks, with different types of nodes representing uncertain quantities, decision variables, deterministic functions, and value models. IDs were first developed in the mid 1970s as a decision analysis tool to offer an intuitive way to identify and display the essential elements, including decisions, uncertainties, and objectives, and how they influence each other. 
In general an ID is a directed acyclic graph with three types of nodes and three types of arcs between nodes. The first is called Decision node, it is drawn as a rectangle and corresponds to some decision to be made. Chance or Uncertainty node is the second type, which is drawn as an oval and represents an uncertainty to be modeled. The third one is the Value node, which is drawn as a hexagon (or octagon) or diamond, and calculates all possible combinations receiving from factors in the modeling environment acting as parent nodes. A Functional arc ends at a value node and represents the contribution of the node at its tail to the calculated value. The second type of arc is the Conditional, which ends at a chance node and indicates that the uncertainty at its head is probabilistically related to the node (oval) at its tail. Finally, an Informational arc ends at a decision node and indicates that the decision at its head is made according to the outcome of the node at its tail, which is known beforehand. A simple example of an ID is presented in Figure 1.

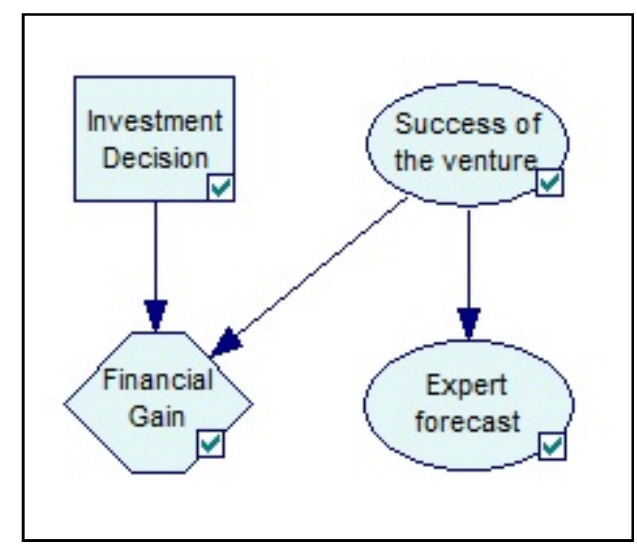

Fig. 1. A simple Influence Diagram

\section{Modeling the Cloud Adoption Process}

\subsection{Model Design}

Modeling the Cloud adoption decision-making process was implemented combining two methods: (i) Literature study and (ii) Collection of expert opinion through specially prepared questionnaires followed by interviews. More specifically, a smallscale literature review on the subject was conducted in order to identify a number of factors that potentially influence such a decision which would then be used to form the nodes of our model. The next step involved identifying a group of three experts with strongly related background to the subject (i.e. Cloud Computing related positions). An initial list of factors was then prepared and the experts were asked to evaluate the list and prompted to add or remove factors based on their expertise and working experience. Finally, one more round of discussion with experts was conducted in order to finalize the list of factors. These factors were used to form the nodes of the ID model and are listed in Table 1. 
Table 1. Factors Influencing Cloud Adoption

\begin{tabular}{|c|c|c|}
\hline Name & Definition & Reference \\
\hline Legal Issues & $\begin{array}{l}\text { Cloud adoption compliance with all legislative } \\
\text { issues. Ability to adjust when legal requirements } \\
\text { grow. }\end{array}$ & {$[23],[17],[18],[25]$} \\
\hline Availability & $\begin{array}{l}\text { The amount of time that Cloud Service(s) is } \\
\text { operating as the percentage of total time it should } \\
\text { be operating. }\end{array}$ & $\begin{array}{l}{[14],[9],[15],[10],} \\
{[23],[18]}\end{array}$ \\
\hline Security & $\begin{array}{l}\text { Security of service: data transfer, data stores, web } \\
\text { servers, web browsers. }\end{array}$ & $\begin{array}{l}{[4],[14],[9],[15],} \\
{[16],[10],[23],[17],} \\
{[18],[25]}\end{array}$ \\
\hline Cost / Pricing & $\begin{array}{l}\text { Operational - running costs, migration costs etc. } \\
\text { Cost benefits from Cloud adoption. }\end{array}$ & $\begin{array}{l}{[4],[13],[14],[9],} \\
{[15],[16],[24],[10],} \\
{[23],[25],[26,][27]}\end{array}$ \\
\hline ROI & Return on Investment. & {$[13],[9],[16]$} \\
\hline Compliance & Business and Regulatory compliance. & {$[9],[10],[23],[18]$} \\
\hline Performance/Processing & $\begin{array}{l}\text { Does Cloud adoption perform the process to the } \\
\text { desired quality? }\end{array}$ & $\begin{array}{l}{[14],[9],[15],[16],} \\
{[10],[23]}\end{array}$ \\
\hline Scalability & $\begin{array}{l}\text { Ability to meet an increasing workload require- } \\
\text { ment by incrementally adding a proportional } \\
\text { amount of resources capacity. }\end{array}$ & {$[15],[17]$} \\
\hline Privacy/ Confidentiality & Privacy and confidentiality coverage. & $\begin{array}{l}{[4],[13],[9],[18],} \\
{[25],[22]}\end{array}$ \\
\hline Elasticity & $\begin{array}{l}\text { Ability to commission or decommission resource } \\
\text { capacity on the fly. }\end{array}$ & {$[14],[9],[25]$} \\
\hline $\begin{array}{l}\text { Data Access / Import- } \\
\text { Export }\end{array}$ & Access to data in various ways. & {$[15],[18],[25]$} \\
\hline Technology Suitability & $\begin{array}{l}\text { Does Cloud technology exhibit the appropriate } \\
\text { technological characteristics to support the pro- } \\
\text { posed SaaS? }\end{array}$ & {$[14],[9],[25]$} \\
\hline Hardware Access & $\begin{array}{l}\text { Degree of Cloud Service accessibility, on local } \\
\text { hardware. }\end{array}$ & {$[14],[9]$} \\
\hline Audit ability & $\begin{array}{l}\text { Ability of Cloud service to provide access and } \\
\text { ways for audit. }\end{array}$ & [15] \\
\hline
\end{tabular}

Considering the influencing factors that were extracted and processed as described above, we proceeded with the development of the ID shown in Figure 2 using the GeNIe toolbox [3]. The nodes representations and their dependencies are able to model our question and provide a final decision node. 


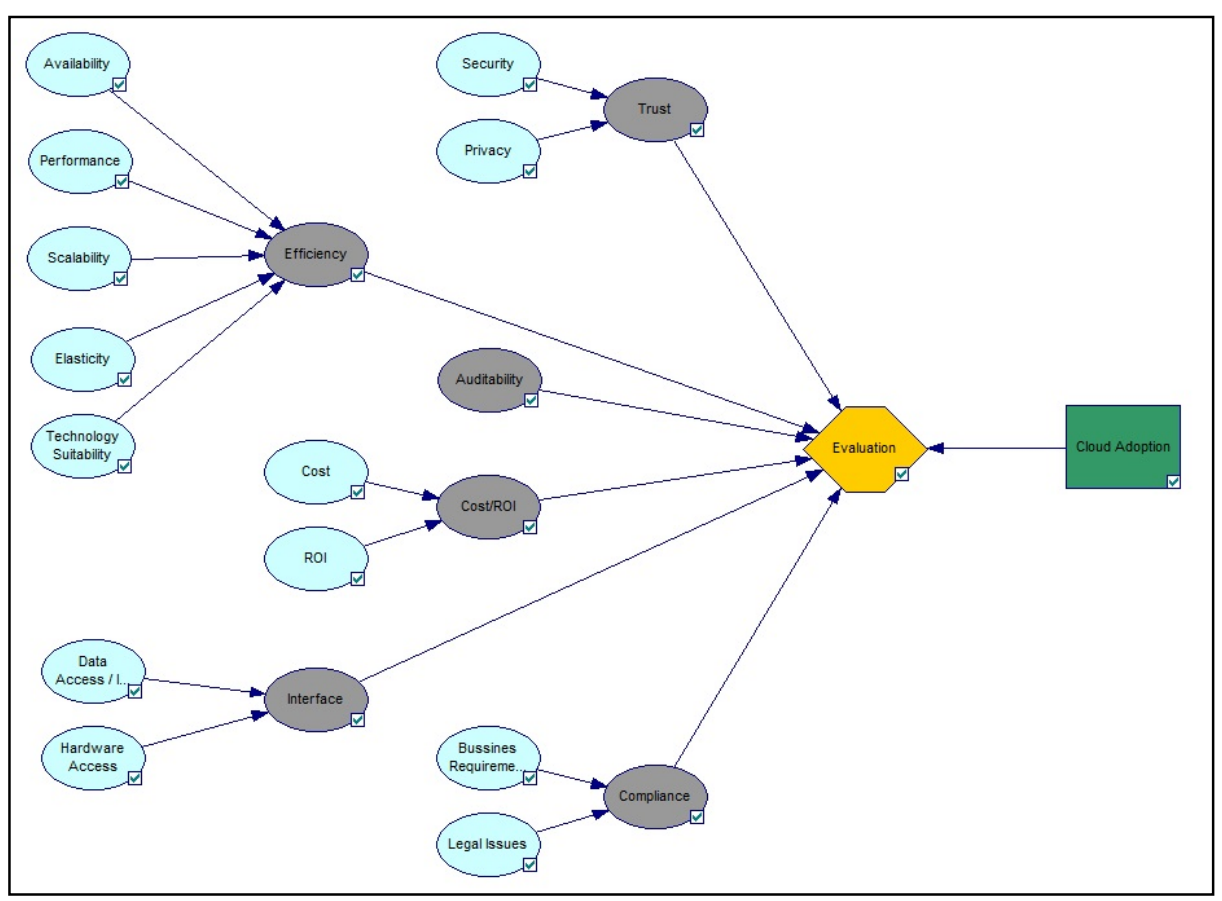

Fig. 2. “Adopt Cloud or Not” Influence Diagram

\section{2 $\quad$ Experimental Results}

Aiming to test and evaluate the performance of the proposed model, two hypothetical scenarios were first conducted representing the so called "extreme cases", that is, a situation where everything would be in favor of Cloud adoption (positive scenario) and the opposite case (negative scenario). The target was to have a reasonable answer by the evaluation node under known situations and assess the performance of the model demonstrating that the model behaves correctly and as expected to. Next, the model was tested on a number of real-world scenarios, that is, cases collected from real customers of two international Cloud services providers with the aid of the same experts that were utilized to construct our model. The two extreme scenarios and the real-world cases experimentation are described below. Table 2 presents the input values to the model that describe the current situation at the point of time when the decision was about to be made for each of the four scenarios tested expressed in, linguistic terms And transformed to their numerical counterparts. 
Table 2. Input values for the four scenarios tested

\begin{tabular}{|c|c|c|c|c|c|}
\hline Factor & Term & Positive & Negative & Real 1 & Real 2 \\
\hline \multirow[t]{3}{*}{$\overline{\text { Legal }}$} & High & 0.8 & 0 & 0.6 & 0.2 \\
\hline & Medium & 0.2 & 0.2 & 0.3 & 0.6 \\
\hline & Low & 0 & 0.8 & 0.1 & 0.2 \\
\hline \multirow[t]{3}{*}{ Availability } & High & 0.8 & 0 & 0.6 & 0.2 \\
\hline & Medium & 0.2 & 0.2 & 0.3 & 0.6 \\
\hline & Low & 0 & 0.8 & 0.1 & 0.2 \\
\hline \multirow[t]{3}{*}{ Security } & High & 0.8 & 0 & 0.6 & 0.2 \\
\hline & Medium & 0.2 & 0.2 & 0.3 & 0.6 \\
\hline & Low & 0 & 0.8 & 0.1 & 0.2 \\
\hline \multirow[t]{3}{*}{ Cost / Pricing } & High & 0 & 0.8 & 0.3 & 0.7 \\
\hline & Medium & 0.2 & 0.2 & 0.6 & 0.3 \\
\hline & Low & 0.8 & 0 & 0.1 & 0 \\
\hline \multirow[t]{3}{*}{ ROI } & High & 0.8 & 0 & 0.3 & 0 \\
\hline & Medium & 0.2 & 0.2 & 0.6 & 0.2 \\
\hline & Low & 0 & 0.8 & 0.1 & 0.8 \\
\hline \multirow[t]{3}{*}{ Compliance } & High & 0.8 & 0 & 0.6 & 0 \\
\hline & Medium & 0.2 & 0.2 & 0.3 & 0.2 \\
\hline & Low & 0 & 0.8 & 0.1 & 0.8 \\
\hline \multirow{3}{*}{ Performance/Processing } & High & 0.8 & 0 & 0.3 & 0 \\
\hline & Medium & 0.2 & 0.2 & 0.6 & 0.2 \\
\hline & Low & 0 & 0.8 & 0.1 & 0.8 \\
\hline \multirow[t]{3}{*}{ Scalability } & High & 0.8 & 0 & 0.8 & 0 \\
\hline & Medium & 0.2 & 0.2 & 0.2 & 0.6 \\
\hline & Low & 0 & 0.8 & 0 & 0.4 \\
\hline \multirow[t]{3}{*}{ Privacy/Confidentiality } & High & 0.8 & 0 & 0.3 & 0 \\
\hline & Medium & 0.2 & 0.2 & 0.6 & 0.2 \\
\hline & Low & 0 & 0.8 & 0.1 & 0.8 \\
\hline \multirow[t]{3}{*}{ Elasticity } & High & 0.8 & 0 & 0.8 & 0 \\
\hline & Medium & 0.8 & 0 & 0.2 & 0.6 \\
\hline & Low & 0.2 & 0.2 & 0 & 0.4 \\
\hline Data Access / Import- & High & 0 & 0.8 & 0.6 & 0 \\
\hline \multirow[t]{2}{*}{ Export } & Medium & 0.8 & 0 & 0.3 & 0.6 \\
\hline & Low & 0.2 & 0.2 & 0.1 & 0.4 \\
\hline \multirow[t]{3}{*}{ Technology Suitability } & High & 0 & 0.8 & 0.6 & 0 \\
\hline & Medium & 0.8 & 0 & 0.3 & 0.6 \\
\hline & Low & 0.2 & 0.2 & 0.1 & 0.4 \\
\hline \multirow[t]{3}{*}{ Hardware Access } & High & 0 & 0.8 & 0.3 & 0.2 \\
\hline & Medium & 0 & 0.8 & 0.6 & 0.8 \\
\hline & Low & 0.2 & 0.2 & 0.1 & 0 \\
\hline \multirow[t]{3}{*}{ Auditability } & High & 0.8 & 0 & 0.3 & 0 \\
\hline & Medium & 0.8 & 0 & 0.6 & 0.6 \\
\hline & Low & 0.2 & 0.2 & 0.1 & 0.4 \\
\hline
\end{tabular}

\section{Scenario 1: Positive Case}

This case assumes an ideal environment where the Cloud services offered perfectly match a customer's needs. Thus, the values for each leaf node were chosen so that they reflect this ideal setting and guide the evaluation node to a positive value. In this scenario the diagram executed and the values on the evaluation node were calculated to 0.77 for "Yes" and 0.23 for "No". This means that the model correctly recognized the positive environment and suggested that a decision in favor of Cloud adoption should be taken based on the values "read" in the nodes and the current influences between them. 


\section{Scenario 2: Negative Case}

Working in the same way as with the positive scenario, appropriate values for each leaf node were chosen this time to guide the evaluation node to a negative value. Executing the model using the values for the negative scenario values the evaluation node yielded 0.75 for "No" and 0.25 for "Yes" which perfectly matched the expected behavior.

By using the above "extreme" scenarios it became evident that the proposed model behaves successfully as it recognized correctly the conditions of the environment and predicted the right decision. Therefore, we may now proceed to test its performance on real-world scenarios.

\section{Real-World Scenarios}

As mentioned earlier, we identified two different cases, with the help of Cloud providers. One customer who decided to proceed with Cloud adoption and one customer who rejected it. In order to be able to retrieve the leaf nodes values for each case separately we had a series of interviews both with the Cloud providers and the customers. We managed to record and adjust these values, which essentially reflected the state of the offered service and the associated factors describing each customer's particular situation at the moment of decision.

The first case involved an academic institution with a medium to large size which requested a comprehensive solution for email services. In that case easily someone can discern from the input values that the conditions were in favor of a positive decision. The second case involved a medium insurance broker organization which requested a complete email Cloud package and also a Cloud infrastructure to fit a heavy tailored made owned system. In that case, someone can hardly make an assessment of the final decision, based on the input values.

By executing the proposed model on these two real world scenarios, the results given in Table 3 were produced which were compared with the real decisions. In both cases the model successfully predicted the right decision.

Table 3. Model's decisions compared with real decisions

\begin{tabular}{ccc}
\hline Real Scenario & $\begin{array}{c}\text { ID model's decision } \\
\text { (Evaluation Node Values) }\end{array}$ & Real decision \\
\hline A & Yes $(0.61)$ & Yes \\
B & No $(0.74)$ & No \\
\hline
\end{tabular}

\section{Conclusions}

Although Cloud Computing has gone from infancy to a new mature state, customers are still facing many challenges with respect to its adoption. The study and understanding of various parameters such as benefits and problems that are involved in this transition is far from an easy and straightforward procedure. This paper proposed a new approach, aiming to assist the Cloud adoption decision process. We demonstrated 
how a new model based on Influence Diagrams can be constructed and applied to face decision making on the Cloud adoption problem.

The proposed model was experimentally evaluated initially on two extreme scenarios, an ideal setting in favor of Cloud adoption and a completely negative leading to Cloud rejection, showing successful performance. This enabled further experimentation with two real-world scenarios collected form experts/developers in the local software industry. The model succeeded in matching its estimation with the corresponding real decisions.

Although the results may be considered quite encouraging, there are quite a few enhancements that may be performed the model so as to fit the problem more accurately. Further research steps can be separated in two groups. The first involves enriching the knowledge regarding Cloud Computing and its parameters and the second the optimization of the decision tool. The speed with which Cloud Computing and its corresponding technology evolves necessitates the continuous study of the model. In addition, more real-world case scenarios could give a helpful feedback for better calibration of the model and finally, possible expansion and re-identification of the diagram will be investigated so as to include more nodes representing better the real Cloud environment.

\section{References}

1. Shachter, R.D.: Evaluating Influence Diagrams. Operations Research 34(6), 871-882 (1986)

2. Howard, R.A.: Readings on the Principles and Applications of Decision Analysis: Professional collection, vol. 2. Strategic Decisions Group (1984)

3. Laboratory, D.S.: Graphical Network Interface (GeNie). (University of Pittsburgh)

4. GeNIe\&SMILE (2013), http: / / genie.sis . pitt. edu (retrieved January 15, 2013)

5. Khajeh-Hosseini, A., Sommerville, I., Sriram, I.: Research challenges for enterprise Cloud Computing. arXiv preprint arXiv:1001.3257 (2010)

6. Papatheocharous, E., Trikomitou, D., Yiasemis, P., Andreou, S.A.: Cost Modelling and estimation in agile software development environments using influence diagrams. In: 13th International Conference on Enterprise Information Systems (ICEIS(3)), Beijing, pp. 117-127 (2011)

7. Wu, W.-W.: Developing an explorative model for SaaS adoption. Expert Systems with Applications 38, 15057-15064 (2011)

8. Wu, W.-W.: Mining significant factors affecting the adoption of SaaS using the rough set approach. The Journal of Systems and Software 84, 435-441 (2011)

9. Mell, P., Grance, T.: The NIST Definition of Cloud Computing. National Institute of Standards and Technology (2009)

10. Khajeh-Hosseini, A., Greenwood, D., Smith, J.W., Sommerville, I.: The Cloud Adoption Toolkit: supporting Cloud adoption decisions in the enterprise. Software: Practice and Experience 42(4), 447-465 (2012)

11. Kim, W., Kim, S.D., Lee, E., Lee, S.: Adoption issues for Cloud Computing. In: MoMM 2009, pp. 2-5 (2009)

12. Wu, W.W., Lan, L.W., Lee, Y.T.: Exploring decisive factors affecting an organization's SaaS adoption: A case study. International Journal of Information Management 31(6), 556-563 (2011) 
13. Gabus, A., Fontela, E.: World problems, an invitation to further thought within the framework of DEMATEL. BATTELLE Institute, Geneva Research Centre, Geneva, Switzerland (1972)

14. Yang, H., Tate, M.: Where are we at with Cloud Computing?: a descriptive literature review (2009)

15. Greenwood, D., Khajeh-Hosseini, A., Smith, J.W., Sommerville, I.: The Cloud adoption toolkit: Addressing the challenges of Cloud adoption in enterprise. Technical Report (2010)

16. Armbrust, M., Fox, A., Griffith, R., Joseph, A.D., Katz, R., Konwinski, A., Zaharia, M.: A view of Cloud Computing. Communications of the ACM 53(4), 50-58 (2010)

17. Benlian, A., Hess, T.: Opportunities and risks of software-as-a-service: Findings from a survey of IT executives. Decision Support Systems 52(1), 232-246 (2011)

18. Godse, M., Mulik, S.: An approach for selecting software-as-a-service (SaaS) product. In: IEEE International Conference on Cloud Computing, CLOUD 2009, pp. 155-158. IEEE (2009)

19. Yang, J., Chen, Z.: Cloud Computing Research and security issues. In: 2010 International Conference on Computational Intelligence and Software Engineering (CiSE), pp. 1-3. $\operatorname{IEEE}(2010)$

20. Grandon, E.E., Pearson, J.M.: Electronic commerce adoption: an empirical study of small and medium US businesses. Information \& Management 42(1), 197-216 (2004)

21. de Assunção, M.D., di Costanzo, A., Buyya, R.: A cost-benefit analysis of using Cloud Computing to extend the capacity of clusters. Cluster Computing 13(3), 335-347 (2010)

22. Dash, D., Kantere, V.: \&Ailamaki, A (2009, An economic model for self-tuned Cloud caching. In . In: IEEE 25th International Conference on Data Engineering, ICDE 2009, pp. 1687-1693. IEEE (2009)

23. Pearson, S.: Taking account of privacy when designing Cloud Computing services. In: ICSE Workshop on Software Engineering Challenges of Cloud Computing, CLOUD 2009, pp. 44-52. IEEE (2009)

24. Dillon, T., Wu, C., Chang, E.: Cloud computing: issues and challenges. In: 24th IEEE International Conference on Advanced Information Networking and Applications (AINA), pp. 27-33. IEEE (2010)

25. Bibi, S., Katsaros, D., Bozanis, P.: Application development: Fly to the clouds or stay inhouse? In: 2010 19th IEEE International Workshop on Enabling Technologies: Infrastructures for Collaborative Enterprises (WETICE), pp. 60-65. IEEE (2010)

26. Microsoft Study: Drivers \& Inhibitors to Cloud Adoption for Small and Midsize Businesses, http: / / www.microsoft.com/en-us/news/presskits / telecom/docs/SMBCloud.pdf

27. de Assunção, M.D., di Costanzo, A., Buyya, R.: A cost-benefit analysis of using cloud computing to extend the capacity of clusters. Cluster Computing 13(3), 335-347 (2010) 